\title{
ESTÁGIO SUPERVISIONADO NA ODONTOLOGIA: VIVÊNCIA DA PROMOÇÃO DA SAÚDE E INTEGRAÇÃO MULTIPROFISSIONAL
}

\author{
Supervised Training in Dentistry: experience of health promotion and multidisciplinary \\ integration
}

\section{Prácticas en odontología: vivencia de la promoción de la salud e integración multiprofesional}

\author{
Marina Viudes Bruder \\ Universidade Estadual de Maringá - UEM - Maringá (PR) - Brasil \\ Luiz Fernando Lolli \\ Universidade Estadual de Maringá - UEM - Maringá (PR) - Brasil
}

Ana Rosa Palácios

Secretaria Municipal de Saúde Maringá - Maringá (PR) - Brasil

Najara Barbosa da Rocha

Universidade Estadual de Maringá - UEM - Maringá (PR) - Brasil

\section{Vanessa Cristina Veltrini}

Universidade Estadual de Maringá - UEM - Maringá (PR) - Brasil

André Gasparetto

Universidade Estadual de Maringá - UEM - Maringá (PR) - Brasil

Mitsue Fujimaki

Universidade Estadual de Maringá - UEM - Maringá (PR) - Brasil

\section{RESUMO}

Objetivo: Relatar a vivência nos estágios supervisionados em Saúde Coletiva por acadêmicos de Odontologia. Síntese dos dados: Relato de experiência a partir da vivência das atividades pelos alunos de estágio supervisionado do curso de Odontologia durante os eventos "Espaço Saúde", realizados no período de 2011 a 2015, que ocorreram nas Unidades Básicas de Saúde (UBS). As turmas eram divididas em grupos na UBS e as atividades educativas, realizadas com as crianças, jovens e adultos, eram desenvolvidas pelos alunos com a supervisão de docentes do Departamento de Odontologia da Universidade Estadual de Maringá, Brasil. O município de Maringá desenvolve atividades de promoção da saúde por meio do Programa Maringá Saudável, incluindo neste programa eventos denominados "Espaço Saúde", organizados pelos profissionais das UBS, com finalidade de interagir com a comunidade. Nesses eventos, os acadêmicos de Odontologia realizam diagnóstico de doenças bucais, instrução de higiene bucal e atividades lúdico-educativas para crianças, além de conhecer os demais serviços da UBS. A partir dessa experiência, percebeu-se um despertar para um novo olhar sobre o papel do profissional que trabalha com saúde, por parte da comunidade, do serviço e academia. Conclusão: A integração ensino-serviço-comunidade beneficia a comunidade local, fortalece o serviço e melhora a formação dos acadêmicos. Desperta também a consciência para promoção da saúde e prevenção das doenças, necessária para a melhoria na qualidade de vida da população.

Descritores: Sistema Único de Saúde; Estágio Clínico; Atenção Primária à Saúde, Odontologia.

\section{ABSTRACT}

Objective: To report Dentistry students' experience in supervised training in Public Health. Data Synthesis: Experience report of activities carried out in events called "Health Space" (Espaço Saúde) by Dentistry students under supervised training. The activities took place in Primary Health Care Centers (Unidades Básicas de Saúde - UBS) from 2011 to 2015. The classes were divided into groups and the activities were carried out with children, youth and adults by students under supervision of the faculty of the Department of Dentistry of the State University of Maringá (Universidade Estadual de Maringá), located in the state of Paraná, Brazil. The municipality of Maringá develops health promotion activities through the Healthy Maringá Program (Programa Maringá Saudável), which includes events called "Health Space" organized by UBS professionals with the aim of interacting with the community. In these events, the Dentistry students diagnose oral diseases, teach oral hygiene, and carry out playful educational activities with children; in addition, they get to know the other services offered in the UBS. This experience provided a new understanding of the role of the health professional by the community, the service, and 
the university. Conclusion: The teaching-service-community integration benefits the local community, strengthens the service, and improves the training of students. It also raises awareness for health promotion and disease prevention, which is necessary to improve the quality of life of the population.

Descriptors: Unified Health System; Clinical Clerkship; Primary Health Care, Dentistry.

\section{RESUMEN}

Objetivo: Relatar la vivencia de las prácticas de Salud Colectiva de académicos de Odontología. Síntesis de los datos: Relato de experiencia a partir de la vivencia de las actividades de los alumnos de prácticas del curso de Odontología durante los eventos "Espacio Salud" realizados en el periodo entre 2011 y 2015 en las Unidades Básicas de Salud (UBS). Las clases fueron divididas en grupos en la UBS y las actividades educativas realizadas con los niños, jóvenes y adultos fueron desarrolladas por los alumnos bajo la supervisión de los docentes del Departamento de Odontología de la Universidad Estadual de Maringá, Paraná, Brasil. El municipio de Maringá desarrolla actividades de promoción de la salud a través del Programa Maringá Saludable que incluye en este programa eventos denominados "Espacio Salud" que son organizados por los profesionales de las UBS con el objetivo de interaccionar con la comunidad. En los eventos los académicos de Odontología realizan el diagnóstico de enfermedades de la boca, promueven la explicación sobre la higiene bucal y actividades lúdicoeducativas para niños además de conocer los otros servicios de la UBS. A partir de esa experiencia, hubo un despertar para una nueva mirada sobre el papel del profesional sanitario de parte de la comunidad, del servicio e de la academia. Conclusión: La integración enseñanza-servicio-comunidad conlleva beneficio para la comunidad local, fortalece el servicio y mejora la formación de los académicos. Además, despierta la consciencia para la promoción de la salud y la prevención de enfermedades que son necesarias para la mejoría de la calidad de vida de la población.

Descriptores: Sistema Único de Salud; Prácticas Clínicas; Atención Primaria de Salud, Odontología.

\section{INTRODUÇÃO}

Os estágios supervisionados são considerados espaços no curso de graduação que permitem integrar o aluno ao contexto social e econômico da região de atuação, nos quais são realizados trabalhos que vão desde a educação em saúde até a reversão dos danos causados pelas doenças ${ }^{(1)}$.

Apesar de a saúde ser um direito fundamental do ser humano, conforme traz a Constituição Federal de $1988^{(2)}$, os estágios supervisionados em cursos de graduação ainda dão pouca ênfase às experiências que oportunizam a vivência da prática da promoção da saúde ${ }^{(3)}$. A grande maioria das atividades práticas, dentro do curso de Odontologia, ainda foca principalmente no tratamento curativo ${ }^{(4)}$.

As diretrizes curriculares nacionais para os cursos de Odontologia ressaltam a importância das ações coletivas contextualizadas, além da formação técnica, resultando na formação de profissionais habilitados a prestar atenção integral e humanizada à população. Também destacam como salutares o trabalho em equipe e o desenvolvimento de ações que visam a promoção da saúde e fortalecimento da atenção primária ${ }^{(5)}$.

Atualmente, muitos profissionais liberais estão buscando a estabilidade dos empregos públicos porque têm tido dificuldades para se estabelecerem no competitivo mercado de trabalho odontológico. Verifica-se, porém, que muitas são as limitações encontradas no perfil de formação do cirurgião-dentista. Em geral, não atende às necessidades requeridas de um trabalhador do serviço público ${ }^{(6-8)}$, por isso, a articulação entre as Instituições de Ensino Superior (IES) e o Sistema Único de Saúde (SUS) tem se mostrado fundamental para que o próprio sistema possa ordenar a formação de recursos humanos em saúde e a graduação possa trabalhar em sinergia no desenvolvimento das competências necessárias para a consolidação do SUS ${ }^{(9)}$. Essas necessidades tem sido pensadas e implementadas pelas IES, particularmente nos estágios supervisionados ${ }^{(1)}$, cujos objetivos são impulsionar a relação ensino-serviço-comunidade, ampliar as relações da universidade com a sociedade e colocar o futuro profissional em contato com as diversas realidades ${ }^{(1,10)}$.

O curso de Odontologia da Universidade Estadual de Maringá (UEM), Paraná, Brasil, busca, desde sua criação, em 1988, formar profissionais adequados à necessidade do mercado de trabalho e preparados para atuarem no sistema de saúde vigente no país. Em 1992, implementou mudanças no projeto pedagógico baseadas em um currículo integrado, já contando com uma carga horária de estágio totalizando $20 \%$ do total do curso, ou seja, 1.015 horas de atividades extramurais. Essas mudanças foram consideradas inovadoras e já se encontravam em consonância com as Diretrizes Curriculares Nacionais (DCN) para os cursos de graduação em Odontologia, publicadas posteriormente em $2002^{(11)}$. Em 1994, o curso contou com a abertura de um campo de aprendizado na rede municipal de saúde em Maringá, que desde então tem representado uma parceria importante para que os graduandos vivenciem a prática e a realidade do SUS.

Várias propostas e iniciativas vêm sendo experimentadas e implementadas ao longo dos últimos anos, visando a formação do aluno de graduação na perspectiva do SUS, particularmente impulsionadas por políticas intersetoriais dos Ministérios da Saúde e do Ministério da Educação, como o Programa Nacional de Reorientação da Formação Profissional em Saúde (PróSaúde) e o Programa de Educação pelo Trabalho para a Saúde (PET-Saúde) ${ }^{(12)}$. 
O Departamento de Odontologia da UEM, a partir da Portaria Interministerial $\mathrm{n}^{\mathrm{0}}$ 2.101/2005, participou da primeira etapa do Pró-Saúde, que constituiu um importante marco inspirador de mudanças de pensamentos e práticas, bem como o fortalecimento da tríade ensino/serviço/comunidade. Apesar das dificuldades e dos grandes desafios, o desenvolvimento do programa oportunizou a criação de uma massa crítica de docentes engajados com o movimento de mudança na formação dos profissionais de saúde, e as ações, desde então, têm sido planejadas e executadas de forma colegiada ${ }^{(13)}$. Em 2009, por meio da Portaria Interministerial n 3/2009, o curso de Odontologia da UEM iniciou sua participação no Projeto PET-Saúde, que funciona vinculado a diferentes UBS e diversos atores, os quais vivenciam na prática a multiprofissionalidade, a interdisciplinaridade e a integralidade no cuidado à saúde, proporcionando uma formação diferenciada, interprofissional, pela vivência da realidade do SUS e da integração ensino-serviço-comunidade na construção de um sistema mais humanizado, resolutivo e unificado ${ }^{(14)}$.

Os estágios curriculares supervisionados em Saúde Coletiva, no curso de Odontologia da UEM, são realizados no $2^{\circ}$ ano, com 17 horas; no $3^{\circ}$ ano, com 34 horas; no $4^{\circ}$ ano, com 204 horas; e no $5^{\circ}$ ano, com 760 horas. Em 2007, com a necessidade da inclusão de atividades de territorialização e atendimento clínico para os alunos do $5^{\circ}$ ano, foi criada a "Clínica do Programa de Saúde da Família (PSF)", a qual passou a ser denominada "Clínica Ampliada" a partir de 2009. A Clínica Ampliada está inserida dentro do Estágio Supervisionado em Saúde Coletiva IV e sua operacionalização segue os pressupostos da Política Nacional de Humanização, que visa o atendimento integral às necessidades e tem como objetivo produzir saúde e autonomia aos indivíduos e comunidade ${ }^{(15)}$. Dessa maneira, o curso de Odontologia busca diversificar os cenários de prática e inserir os alunos em vivências de diagnóstico e planejamento individual e coletivo, de promoção de saúde e atendimento clínico odontológico, sendo todas essas consideradas importantes para o desenvolvimento de habilidades e competências para o bom exercício profissional.

Em 1974, foi lançado o conceito de promoção de saúde, que mostrou sua relevância na organização dos serviços de saúde pública. Esses preceitos foram reforçados e entendidos pela declaração de Alma-Ata, em 1978 ${ }^{(16)}$. A primeira Conferência Internacional de Promoção de Saúde, realizada em Otawa (1986) ${ }^{(17)}$, deu uma definição mais ampla de saúde, envolvendo educação, mudança de políticas públicas, enfoque ambientalista e ação comunitária. A carta de Otawa ${ }^{(17)}$ define a promoção de saúde como sendo o procedimento que visa aumentar a capacidade dos indivíduos e das comunidades de controlarem a sua saúde, no sentido de melhorar.

Para atingir um estado de completo bem-estar físico, mental e social, o indivíduo, ou o grupo, deve estar preparado a identificar e realizar as suas pretensões, corresponder as suas faltas ou as modificarem, adequando-se ao meio ${ }^{(17)}$. A partir da Agenda 21, resultante da Conferência das Nações Unidas sobre o Meio Ambiente e o Desenvolvimento, que aconteceu no Rio de Janeiro, em 1992, a promoção de saúde também ficou definida como um instrumento de planejamento para a construção de sociedades mais sustentáveis, em diferentes bases geográficas, incluindo métodos de proteção ambiental, justiça social e eficiência econômica. Esse conceito, portanto, une-se ao de Cidade Saudável, da Organização Mundial da Saúde ${ }^{(18)}$.

O estágio curricular é uma modalidade de ensino obrigatória no curso de graduação em Odontologia, que tem o escopo de propiciar aos discentes do curso uma visão de sua profissão de forma ampla e concreta. O estudo é relevante para a Saúde Coletiva, pois tem como foco discutir aspectos da formação dos futuros profissionais, podendo-se ressaltar a obtenção de uma visão mais generalista da situação de saúde da população, focada na promoção da saúde.

Diante desse contexto, o presente estudo tem como objetivo relatar a vivência nos estágios supervisionados em Saúde Coletiva por acadêmicos de Odontologia.

\section{SÍNTESE DOS DADOS}

Trata-se de um de relato de experiência, a partir da vivência das atividades dos alunos da graduação do curso de Odontologia na disciplina Estágio Supervisionado em Saúde Coletiva, da Universidade Estadual de Maringá, Paraná, Brasil, no período de 2011 a 2015. Baseado no fortalecimento das comunidades por meio da educação em saúde, o município de Maringá criou em 2006 o "Programa Maringá Saudável", que segue as diretrizes da "Rede de Municípios e Comunidades Saudáveis", uma iniciativa da Organização Pan-Americana da Saúde (OPAS) associada à Organização Mundial de Saúde ${ }^{(18)}$. Nesse programa pode-se citar algumas estratégias que têm sido desenvolvidas, como o "Espaço Saúde", cujo objetivo é praticar educação em saúde, prevenindo doenças e oferecendo autonomia, trazendo melhorias na qualidade de vida dos membros da comunidade. Essas atividades têm estabelecido, ao longo dos anos, parcerias com instituições de ensino superior e técnico, organizações não governamentais $(\mathrm{ONG})$, secretarias municipais, conselhos locais de saúde, associações de bairro, artesãos e empresas locais ${ }^{(19)}$. A participação dos alunos deu-se sob a supervisão do professor orientador em todas as fases do desenvolvimento da experiência, que ocorreu nas Unidades Básicas de Saúde (UBS).

As turmas de alunos foram divididas em grupos e as atividades educativas foram realizadas com as crianças, jovens e adultos que aguardavam atendimento na UBS. Iniciava-se com um convite informal aos usuários e seus acompanhantes para participarem das atividades de sala de espera, enquanto aguardavam atendimento. Então, ocorria a apresentação de todos, adotando-se a proposta de um tema gerador, sendo, algumas vezes, pré-selecionado pelos acadêmicos, pela equipe de saúde e outros. Esse tema gerador era usado para incitar as reflexões e as problematizações, mas, durante várias práticas, eram 
extrapolados, permeando diversos assuntos do cotidiano social e cultural local. Nessa direção, para concretizar os momentos propostos na sala de espera, aplicaram-se vários materiais didáticos para facilitar a troca de saberes entre usuários e acadêmicos.

Para as crianças, realizaram-se atividades lúdicas, de modo a promover a aprendizagem de aspectos mais abrangentes, como a mudança de comportamento e melhoria na qualidade de vida ${ }^{(20)}$. Para isso, foram utilizados fantoches e uma linguagem de fácil entendimento para abordar os cuidados com a saúde bucal e apresentar a figura do cirurgião-dentista como um parceiro da criança, colaborando no cuidado da saúde dos dentes. Também se realizou demonstração de técnicas de escovação dos dentes e utilização de fio dental com a ajuda de macromodelos.

A participação de jovens e adultos foi realizada com diálogos, a partir de painéis ilustrativos que continham informações sobre as principais doenças bucais, como cárie dentária, periodontite, pulpite, câncer bucal, bem como, sobre os cuidados necessários para uma boa saúde bucal, como técnicas adequadas de escovação dental, preservação e higienização das próteses, autoexame bucal e saúde bucal da gestante.

A experiência foi relatada em dois eixos, o primeiro aponta contribuições do projeto para a comunidade pelas atividades realizadas e o segundo em relação ao impacto da prática na formação profissional dos alunos de Odontologia, a partir das observações realizadas fundamentadas na literatura científica.

\section{Contribuições do projeto para a comunidade pelas atividades realizadas}

As atividades realizadas em sala de espera são um momento oportuno de humanização no atendimento, pois possibilitam o diálogo em relação à promoção da saúde. Permitem ao paciente, independentemente de sua faixa de idade, entrar em contato com seus medos, inquietações e demandas. Assim, tem-se como meta a substituição da angústia, quando ocasionada pelo medo da consulta ao dentista, por sentimentos de curiosidade e bem-estar, e por uma maior informação dos profissionais de saúde. Além disso, é uma ocasião que oportuniza a participação dos familiares e acompanhantes, de forma que todos se envolvem no processo, tendo como benefício a diminuição do desgaste físico e emocional e, do mesmo modo, maior rendimento do trabalho profissional $^{(21)}$.

Em um estudo ${ }^{(22)}$, por ocasião dos momentos de sala de espera, os participantes, corroborando com o estudo em questão, também podiam extrair suas dúvidas e discutir sobre diversos temas, sendo um momento importante de aproximação entre o saber popular e o saber técnico/científico gerado na graduação. Outro ponto relevante neste estudo ${ }^{(22)}$ foi o aspecto organizacional e a motivação da equipe de saúde, que lembrava a relevância das salas de espera como estratégia positiva no próprio atendimento dos usuários, pois podiam atender de forma mais tranquila e organizada, a fim de proporcionar mais atenção aos pacientes.

A educação em saúde bucal com crianças, deve ressaltar a relevância de uma escovação realizada com o auxílio de um adulto, expondo a arcada dentária de uma criança, mostrando a importância de manter dentes e tecidos adjacentes saudáveis, como manter a escovação efetiva e como utilizar o fio dental. As atitudes e os hábitos adquiridos durante as primeiras fases da vida serão levados para as fases seguintes, quando se começa a assumir a responsabilidade pelos próprios atos ${ }^{(23)}$. A motivação estabelecida através da educação pode gerar amplo impacto sobre o desempenho da criança. É nesse sentido que a educação em saúde se concretiza como uma estratégia básica nos serviços de saúde, uma vez que busca preparar os indivíduos para conduzir as suas próprias vidas através da educação, em que os sujeitos se afirmam como protagonistas da sua própria história ${ }^{(2)}$.

Em relação aos adultos, a preferência desses usuários, no que diz respeito às exodontias, são construções históricas, agregadas ao cognitivo coletivo, em decorrência de práticas relacionadas à saúde bucal ao longo dos anos. Os usuários, então, pela dificuldade de acesso e/ou pela dificuldade de resolver seus problemas de saúde dentais, optavam por terminar seu sofrimento em relação àquele dente. Assim, a criação de um vínculo com o usuário leva-o a discutir as reais necessidades e responsabilidades, evitando que a decisão do procedimento terapêutico esteja centrada no usuário e que seja realizado simplesmente pela manifestação de sua vontade ${ }^{(24)}$. O vínculo poderá ser construído por ocasião da educação em saúde, e precisará, portanto, interagir com a busca da melhor conduta cuidadora ${ }^{(24)}$.

\section{Impacto da prática na formação profissional dos alunos de Odontologia}

O interesse e a preocupação das instituições de ensino na área da saúde, dos profissionais de saúde, dos usuários, gestores e técnicos dos serviços públicos, permitem que se estabeleçam parcerias que beneficiam a todos. Exemplo disso é o presente relato de experiência sobre a participação no evento "Espaço Saúde", que tem atuado como um facilitador da construção de parcerias e da integração ensino-serviço-comunidade.

Embora as estratégias de educação em saúde tendam a mostrar resultados apenas em longo prazo, pode-se observar, durante o evento, grande interesse do público pelas temáticas abordadas. As crianças participaram ativamente das atividades lúdicas e interagiram no decorrer da apresentação do teatro de fantoches. Os adultos acompanharam atentamente a apresentação dos painéis e fizeram questionamentos. Percebeu-se que a comunidade consegue melhorar a visão do papel do cirurgiãodentista, entendendo que ele não só intervém na doença, mas pode e deve priorizar a prevenção e ajudá-los a ter autonomia para a manutenção de sua saúde.

O estágio supervisionado, para ser bem-sucedido, depende de escolhas acertadas de cenários de prática, de estratégias de condução e de interação ensino-serviço-comunidade, estando sempre voltado para a capacitação de recursos humanos para o 
SUS, com ênfase na prevenção, na educação em saúde e na visão multi e interdisciplinar ${ }^{(3)}$. A participação dos alunos na atividade permitiu que alguns objetivos propostos nas Diretrizes Curriculares Nacionais para o curso de Odontologia, instituídas em 2002, fossem atingidos ${ }^{(25)}$, tais como atenção à saúde, tomada de decisões, comunicação, liderança e educação permanente ${ }^{(9)}$.

A educação permanente é um aspecto importante alcançado, já que o aprendizado contínuo é incorporado ao cotidiano do aluno, que carrega essa dinâmica para a vida profissional, refletindo e analisando criticamente os processos de trabalho de modo a identificar mais facilmente os problemas e as soluções ${ }^{(26)}$.

A partir desse contexto, os alunos perceberam a importância de adquirir habilidades que servirão de base para o processo de desenvolvimento da autonomia e discernimento no oferecer um cuidado integral em saúde com qualidade, eficiência e resolutividade ${ }^{(3,10)}$. Dentre essas habilidades, pode-se destacar o exercício do olhar para a atenção à saúde por meio de ações de prevenção, promoção e proteção, tanto em nível individual quanto coletivo ${ }^{(1)}$. Houve também o aprendizado de interação com outros profissionais da saúde e com o público ${ }^{(9)}$.

As IES devem se preocupar com reformulações curriculares que prevejam a formação de um profissional rico em conhecimentos, habilidades e competências, aptos a decidir e atuar seguramente na educação e prevenção de saúde, atendendo às necessidades sociais ${ }^{(27)}$. Trata-se de tarefa não muito simples, uma vez que os cursos de odontologia ainda se concentram na formação tecnicista ${ }^{(28)}$.

Na prática dos estágios, o graduando se familiariza com a comunidade e com o serviço. Depara-se com a realidade e passa a observar que sua atuação multiprofissional não se resume à cavidade bucal, tampouco à clínica odontológica. Apreende a complexidade em que seu paciente está inserido pelo contato com a realidade do contexto de vida, família e comunidade, e que ele, muitas vezes, está desprovido de informações e condições para o seu próprio cuidado.

Essas afirmações corroboram com os achados de estudo(1) realizado com estudantes de Odontologia sobre o estágio supervisionado para sua formação profissional. Nessa experiência, o aluno também vivenciou a prática do serviço público e passou a conhecer seu papel dentro da equipe da qual fazem parte técnicos e auxiliares em saúde bucal. De certa forma, foi uma antecipação de uma convivência que acontecerá, efetivamente, a partir do quarto ano, quando esse mesmo aluno fará o estágio supervisionado inserido na UBS.

Com a participação no Espaço Saúde, os alunos iniciaram o contato com vivências multiprofissionais. A educação interprofissional ocorre quando discentes de duas ou mais profissões aprendem sobre os outros, com os outros e entre si para possibilitar a colaboração eficaz e melhorar os resultados na saúde ${ }^{(29)}$. Essa oportunidade ofereceu o conhecimento e a compreensão da realidade de outras profissões, além das próprias funções, responsabilidades e aptidões dentro da equipe de saúde. O contexto multiprofissional tem sido trabalhado dentro do curso de Odontologia no estágio supervisionado e foi o início para uma discussão e aproximação do curso para a introdução de uma educação interprofissional dentro do currículo, a fim de formar o aluno para o trabalho em equipe.

Por sua vez, o estágio beneficia o serviço público na medida em que, ao abrir espaço para estagiários, contribui com o treinamento desses futuros profissionais, ajudando a construir um perfil mais adequado às necessidades da população e mais sensibilizado para a educação em saúde. Isso é muito importante, já que o serviço público tem sido o grande empregador de cirurgiões-dentistas. Atualmente, os cirurgiões-dentistas têm deixado a prática privada exclusiva para buscar o vínculo estável do trabalho assalariado. Isso pode ser explicado devido à alta competitividade do mercado odontológico ${ }^{(30)}$.

Apesar da quantidade exacerbada de cirurgiões-dentistas, a situação da saúde bucal no país é preocupante e inspira cuidados, já que grande parte da população ainda não tem acesso aos serviços odontológicos resolutivos. Diante desse quadro, vê-se a necessidade de impulsionar as diferentes frentes de trabalho: a atenção à saúde, a gestão do sistema, a formação dos profissionais e o controle social ${ }^{(8)}$.

Embora o currículo integrado do Departamento de Odontologia na Universidade Estadual de Maringá esteja implantado há mais de 20 anos, sabe-se que o processo de mudança ocorre lentamente. Muitos desafios precisam ser superados e modificações devem ser feitas, tanto na atuação docente quanto discente. Um problema generalizado nos cursos de Odontologia das Instituições de Ensino Superior do país é a supervalorização do profissional especialista em detrimento do generalista ${ }^{(31)}$.

A formação do especialista, incentivada no passado, está ainda disseminada dentro do próprio corpo docente, e isso acaba influenciando a visão dos alunos ${ }^{(32)}$. As instituições de ensino em Odontologia devem formular práticas de ensino capacitadas em formar um cirurgião dentista com conhecimentos, habilidades e competências que permitam decidir e atuar com segurança na prevenção de doenças e na promoção da saúde para atender às necessidades sociais ${ }^{(33)}$, mas que não seja um "operário da odontologia", com práticas puramente tecnicistas ${ }^{(10)}$. Portanto, que tenha uma ampla vivência clínica, saiba utilizar técnicas sofisticadas, preventivas e curativas, sustentada por uma sólida base em ciências básicas. Um profissional com capacidade de interagir com a sociedade, que tenha habilidades de comunicação e liderança, senso crítico, ética e sensibilidade social ${ }^{(33)}$.

Nesse aspecto, a mudança do paradigma biomédico para o modelo holístico, humanista, abrange conteúdos, atitudes e postura dos educadores voltados para a valorização do ser humano, de maneira que o educador deve dar significado ao que leciona, para produzir no discente a vontade pelo conhecimento, tomando atitudes que possibilitem o diálogo entre professor/ discente, de forma que forneçam subsídios para a compreensão da realidade e também para a aplicabilidade das teorias na prática $^{(34)}$. 
A adequação dos cursos da área da saúde às Diretrizes Curriculares Nacionais, juntamente com a realização do estágio supervisionado, contribui para a formação profissional do aluno, capacitando-o para atuar em diferentes ambientes, como o mercado de trabalho, de modo geral, e o serviço público, em especial.

\section{CONCLUSÃO}

A integração ensino-serviço-comunidade beneficia a comunidade local, fortalece o serviço e melhora a formação dos graduandos. Desperta também a consciência para a promoção da saúde e a prevenção das doenças, necessária para melhoria na qualidade de vida da população.

A participação dos alunos, não só do curso de Odontologia da Universidade Estadual de Maringá, como também de outros cursos da área da saúde, no evento "Espaço Saúde", dentro do Estágio Supervisionado, tem sido de grande valia para todos os envolvidos, oferecendo a oportunidade de reconhecer o serviço de saúde como um todo, ter um primeiro contato com a equipe multiprofissional da UBS e um olhar mais apropriado da realidade.

Vale ressaltar que este estudo é um relato de experiência de vivências em Estágio Supervisionado sobre a integração ensino, serviço e comunidade num ambiente de promoção de saúde. Futuros estudos longitudinais sobre a vivência multiprofissional de alunos de graduação na promoção de saúde com amostras mais representativas poderão contribuir para a tomada de decisões sobre o melhor caminho na busca de uma formação profissional de melhor qualidade e que esteja em consonância com as diretrizes curriculares.

\section{REFERÊNCIAS}

1. Cawahisa PT, Terada RSS, Hayacibara MF. Atividades realizadas durante o estágio supervisionado do curso de odontologia da Universidade Estadual de Maringá em um centro de educação infantil. Ciênc Cuid Saúde. 2013;12(2):375-81.

2. Brasil. Constituição da República Federativa do Brasil. São Paulo: Saraiva; 2011.

3. Benito GAV, Tristão KM, Paula ACSF, Santos MA, Ataide LJ, Lima RCD. Desenvolvimento de competências gerais durante o estágio supervisionado. Rev Bras Enferm. 2012;65(1):172-8.

4. Torres LHN, Gibilini C, Mialhe FL, Pereira AC, Meneghim MC, Sousa MLR. Cenários diversificados na formação do aluno de graduação em odontologia. Odontol Clín-Cient. 2013;12(3):203-7.

5. Toassi RFC, Souza JM, Baumgarten A, Rosing CK. Avaliação curricular na educação superior em odontologia: discutindo as mudanças curriculares na formação em saúde no Brasil. Rev ABENO. 2012;12(2):170-7.

6. Ferreira NP, Ferreira AP, Freire MCM. Mercado de trabalho na odontologia: contextualização e perspectivas. Rev Odontol UNESP. 2013;42(4):304-9.

7. Matsumoto SN, Endo MS, Terada RSS, Lolli LF, Hayacibara MF. Features of selective examinations for dentists in Brazil. RGO. 2012;60(2):233-9.

8. Silva ACRM, Garrido TM, Hayacibara MF, Bispo CGC, Silva RL, Morita MC, et al. Perfil de cirurgiões-dentistas formados por um currículo integrado em uma instituição de ensino pública. Rev ABENO. 2012;12(2):147-54.

9. Holanda ICLC, Almeida MM, Hermeto EMC. Indutores de mudança na formação dos profissionais de saúde: pró-saúde e petsaúde. Rev Bras Promoç Saúde. 2012;25(4):389-92.

10. Ministério da Saúde (BR). A aderência dos cursos de graduação em enfermagem, medicina e odontologia às diretrizes curriculares nacionais. Brasília: Ministério da Saúde; 2006.

11. Terada RSS, Nakama L. A implantação das diretrizes curriculares nacionais de odontologia: a experiência de Maringá. Maringá: Hucitec, 2004.

12. Batista SHSS, Jansen B, Assis EQ, Senna MIB, Cury GC. Formação em saúde: reflexões a partir dos Programas Pró-saúde e PET-saúde. Interface Comun Saúde Educ. 2015;19 Supl 1:743-52.

13. Terada RSS, Hayacibara MF, Rigolon CJ, Silva MC, Lolli LF, Hidalgo MM. Implementação do Pró-Saúde no Curso de Odontologia da Universidade Estadual de Maringá. Rev ABENO. 2010;10(2):64-71.

14. Yamakami SA, Albiero ALM, Baesso ML, Fujimaki M, Terada RSS, Pascotto RC. Desenvolvendo o paradigma da integralidade no cuidado à saúde no PET-Saúde/UEM: relato de experiência: atuação de maneira articulada no cuidado integral à saúde da comunidade. Rev ABENO. 2014;14(1):47-56.

15. Hayacibara MF, Lolli FL, Terada RSS, Hidalgo MM, Bispo CGC, Terada HH, et al. Experiência de clínica ampliada em odontologia na Universidade Estadual de Maringá. Rev Bras Educ Med. 2012;36(1 Supl 2):178-83. 
16. Organização Mundial de Saúde. Declaração de Alma-Ata, 1978 [acesso em 2016 Jun 12]. Disponível em: http://cmdss2011. org/site/wp-content/uploads/2011/07/Declara\%C3\%A7\%C3\%A3o-Alma-Ata.pdf

17. Organização Mundial de Saúde. Carta de Ottawa, 1986 [acesso em 2016 Jun 12]. Disponível em: http://bvsms.saude.gov. br/bvs/publicacoes/carta_ottawa.pdf

18. Organização Pan-americana da Saúde. Divisão de promoção e proteção da Saúde. Municípios e comunidades saudáveis - Guia dos prefeitos. Washington: OPAS; 2002 [acesso em 2016 Jun 12]. Disponível em: http://www.bvsde.paho.org/ bvsacd/cd63/MCS_Guiapor/MCS_Guiapor.pdf

19. Sperandio AMG, Veríssimo LCG, Palacios AROP. O processo de desenvolvimento de poíticas públicas saudáveis integradas em rede: a experiência de Maringá-PR. Intellectus. 2011;7(15):10-28.

20. Oliveira JCC. Atividades lúdicas na Odontopediatria: uma breve revisão da literatura. Rev Bras Odontol. 2014;71(1):103-7.

21. Stutz BL, Durighetto IL, Mendonça LC, Gomes SSR, Cunha MJ, Oliveira MLP, et al. Sala de espera em odontologia: uma estratégia para a promoção da saúde bucal e humanização. Extensão. 2012;11(2):162-6.

22. Rosa J, Barth PO, Germani ARM. A sala de espera no agir em saúde: espaço de educação e promoção à saúde. Perspectiva. 2011;35(129):121-30.

23. Valarelli FP, Franco RM, Sampaio CC, Mauad C, Passos VAB, Vitor LLR, et al. Importância dos programas de educação e motivação para saúde bucal em escolas: relato de experiência. Odontol. Clín.-Cient. 2011;10(2):173-6.

24. Santos AM, Assis MMA, Nascimento MAA, Jorge MSB . Vínculo e autonomia na prática de saúde bucal no Programa Saúde da Família. Rev Saúde Pública. 2008;42(3):464-70.

25. Conselho Nacional de Educação (BR), Câmara de Educação Superior. Resolução CNE/CES nº 3, de 19 de fevereiro de 2002. Institui Diretrizes Curriculares Nacionais do Curso de Graduação em Odontologia. Diário Oficial da União, Brasília; 2002.

26. Ministério da Saúde (BR), Secretaria de Gestão do Trabalho e da Educação na Saúde. Departamento de Gestão da Educação em Saúde. Política Nacional de Educação Permanente em Saúde. Brasília: Ministério da Saúde; 2009.

27. Fadel CB, Baldani MH. Percepções de formandos do curso de odontologia sobre as diretrizes curriculares nacionais. Trab Educ Saúde. 2013;11(2):339-54.

28. Warmling AMF, Mello ALSF, Naspolini DS, Canto GL, Souza ER. Contribuições das atividades complementares na formação profissional em odontologia. Rev ABENO 2012;12(2):190-7.

29. Organização Mundial de Saúde. Marco para ação em educação interprofissional e práticas colaborativas. Geneva: OMS; 2010 .

30. Medeiros ACR, Fujimaki M, Higashi DT, Morita MC, Garrido TM, Terada RSS. A qualitative study on the administration and management competencies in a Brazilian dental school. Rev ABENO. 2015;15(4):2-9.

31. Araújo RPC, Mello SMF. Cursos de graduação em odontologia: a formação docente. Pesq Brasil Odontoped Clin Integrada. 2011;11(4):615-25.

32. Toassi RFC, Souza JM, Baumgarten A, Rosing CK. Avaliação curricular na educação superior em odontologia: discutindo as mudanças curriculares na formação em saúde no Brasil. Rev ABENO. 2012;12(2):170-7.

33. Carvalho WM, Cawahisa PT, Scheibel PC, Botelho JN, Terada RSS, Rocha NB, et al. Aceitação da utilização de metodologias ativas nos estágios no SUS por discentes da graduação e pós-graduação. Rev ABENO. 2016;16(1):88-98.

34. Silva RM, Silva ICM, Ravalia RA. Ensino de enfermagem: reflexões sobre o estágio curricular supervisionado. Rev Práxis. 2009;1(1):37-41.

\section{Endereço do primeiro autor:}

Marina Viudes Bruder

Estrada do Galeão, 2121

Bairro: Jardim Guanabara

CEP: 21931-385 - Rio de Janeiro - RJ - Brasil

E-mail: marina@bruder.com.br

\section{Endereço para correspondência:}

Mitsue Fujimaki

Av. Mandacaru, 1550 - Bloco S08

CEP: 87083-170 - Maringá - PR - Brasil

E-mail:mfujimaki@uem.br 